\title{
52. ROTATION EFFECTS IN THE NONGRAVITATIONAL PARAMETERS OF COMETS
}

\author{
Z. SEKANINA \\ Smithsonian Astrophysical Observatory, Cambridge, Mass., U.S.A.
}

\begin{abstract}
The effects of rotation of a cometary nucleus on the character of nongravitational parameters are discussed. It is suggested that the change of a nongravitational acceleration to deceleration (and vice versa) may be related to a precessional motion of the nucleus, or to secular variations in the lag angle coupled with severe orbital modifications.
\end{abstract}

\section{Introduction}

The results of Marsden's $(1969,1970)$ recent dynamical studies have conclusively shown that the inclusion of nongravitational terms in the equations of motion considerably improved the determinacy of the orbits of most short-period comets. It appeared, however, that the 'degree of obedience' to the nongravitational law applied by Marsden varied from comet to comet.

The components of the nongravitational acceleration were assumed to have the form:

$$
\begin{aligned}
& Z_{i}=A_{i} f(r), \quad i=1,2,3, \\
& f(r)=r^{-\alpha} \exp \left(-r^{2} / C\right),
\end{aligned}
$$

where $r$ is the solar distance, $A_{i}$ are constants (nongravitational parameters), $i=1$ for the radial component of the acceleration outward from the Sun, $i=2$ for the transverse component in the orbit plane, perpendicular to the radius vector and in the direction of motion, $i=3$ for the component normal to the orbit plane, and $C$ and $\alpha$ are empirical constants.

In practice the dynamical disobedience of a comet appears in the form of systematic positional residuals within individual apparitions, and it is also reflected in a poorer quality of the nongravitational parameters. It should be pointed out that the deviations from Equations (1) are, on a secular scale, considerably smaller than the deviations from purely gravitational fits even for the most disobedient comets.

\section{Two Groups of Short-Period Comets}

The data on the nongravitational parameters of almost 20 short-period comets determined by Marsden (1969, 1970, 1972), Marsden and Sekanina (1971), and Yeomans (1972a, 1972b) suggest that the comets tend to discriminate into two basic groups.

Group $I$ includes comets with $A_{1}$ always positive, well determined, and $A_{2}$ very small compared to $A_{1}$. Members of this group are $\mathrm{P} /$ Forbes, $\mathrm{P} / \mathrm{Daniel}, \mathrm{P} /$ Faye, $\mathrm{P} / \mathrm{Sch}$ wassmann-Wachmann 2, P/Whipple, and possibly also $\mathrm{P} /$ Schaumasse. $\mathrm{P} /$ Biela and $\mathrm{P} / \mathrm{Pons}-$ Winnecke were likely nineteenth-century members of this group. $\mathrm{P} /$ Honda-MrkosPajdušáková, $\mathrm{P} /$ Giacobini-Zinner, and $\mathrm{P} /$ Borrelly also seem to belong to this group, 
but some of their properties are rather intermediate in character between Groups I and II.

Group II covers comets with $A_{1}$ often negative, badly determined, and with $A_{2}$ comparable or nearly comparable with $A_{1}$ in magnitude. Definite members are P/Encke, $\mathrm{P} /$ Tempel 2, P/d'Arrest, and $\mathbf{P} /$ Wirtanen. Possible members are $\mathrm{P} /$ Tempel-Swift, $\mathrm{P} /$ Arend, and $\mathrm{P} /$ Pons-Winnecke (twentieth century).

There is no difference between the two groups in the degree of the determinacy of $A_{2}$, which is mostly excellent, and in the determinacy of $A_{3}$, which is always poor. Group I can be identified with the obedient comets, the less populated Group II with the unruly ones.

\section{A Model for the Rotation of Cometary Nuclei}

It would be very easy to disregard the small systematic positional deviations from Equations (1) on the grounds that they may be due to a displacement between the center of light and the center of gravity. However, some of the unruly comets show systematic residuals over periods of time during which the geometrical conditions varied considerably, and there should also have been large variations in the relative positions of the optical center and genuine nucleus.

While not denying the existence of occasional effects of photometric displacements on the positional residuals, we do not believe that they can have a dominant influence. We suggest instead that the dynamical disobedience may be due to the approximate character of Equations (1). In the following we compare the empirical nongravitational terms with a simple model for a rotating nucleus.

A simple, yet rather general, model of the nongravitational effects can be developed for a spherical nucleus rotating at a constant angular speed about an axis fixed in space. The momentum of ejected material gives rise to an impulse on the nucleus whose direction can be expressed in terms of the obliquity $\varepsilon$ of the rotation plane to the orbit plane, the longitude $\varphi$ of the subsolar meridian at perihelion from the ascending node of the equator on the orbit, and the lag angle $\Lambda$ of the meridian of effective mass ejection behind the subsolar meridian. We have

$$
\begin{aligned}
& Z_{1}=Z_{0} f(r) a_{1}\left[1-p_{1} \cos 2(\varphi+v)\right], \\
& Z_{2}=Z_{0} f(r) a_{2}\left[1+p_{2} \sin 2(\varphi+v)\right], \\
& Z_{3}=Z_{0} f(r) a_{3} p_{3} \cos (H+\varphi+v),
\end{aligned}
$$

where $v$ is the true anomaly, $Z_{0}$ the magnitude of the impulse (or acceleration),

and

$$
\begin{aligned}
a_{1} & =\left(1-h^{2}\right) \cos 2 H, \\
a_{2} & =\left(1-h^{2}\right) \sin 2 H, \\
a_{1} p_{1} & =a_{2} p_{2}=h^{2} \geqslant 0, \\
a_{3} p_{3} & =2 h\left(1-h^{2}\right)^{1 / 2} \geqslant 0,
\end{aligned}
$$

$$
\begin{aligned}
h & =\sin (\Lambda / 2) \sin \varepsilon \geqslant 0, \\
\tan H & =\tan (\Lambda / 2) \cos \varepsilon .
\end{aligned}
$$


We point out that Equations (2) depend, in contrast to the empirical Equations (1), on the true anomaly: each of the two components in the orbital plane consists of a constant term and a periodic term, whereas the out-of-plane component has a periodic term only.

Equations (2) can be reduced to Equations (1) only if the amplitudes $p_{i}$ of all three periodic terms are zero; then $A_{1}=Z_{0} a_{1}, A_{2}=Z_{0} a_{2}, A_{3}=0$. This would take place if $\varepsilon=0^{\circ}$ or $180^{\circ}$ (rotational axis perpendicular to the orbit plane), or if $\Lambda=0^{\circ}$ (no delay in ejection mechanism). If none of these conditions is at least approximately satisfied, the effect of the periodic terms may result in poor determinacy of the empirical coefficients $A_{i}$.

Thus, the amplitudes $p_{i}$ could have a decisive influence on the 'degree of obedience' of a comet. The greater the $p_{i}$, the more unruly the comet is. We shall now establish the meanings of the amplitudes $p_{i}$ in terms of $\varepsilon$ and $\Lambda$ (they are independent of $\varphi$ ). A plot of $p_{1}$ against $p_{2}$ with $\varepsilon$ and $\Lambda$ as parameters is represented in Figure 1. An extensive analysis, comparing Equations (1) and (2), will appear in full detail elsewhere. Here we point out that, as a result, it is found that the lag angle $\Lambda$ basically determines the degree of accuracy with which $A_{i}$ coefficients can be derived when the periodic terms are neglected. The lag angle is also dominant in controlling the magnitude of the

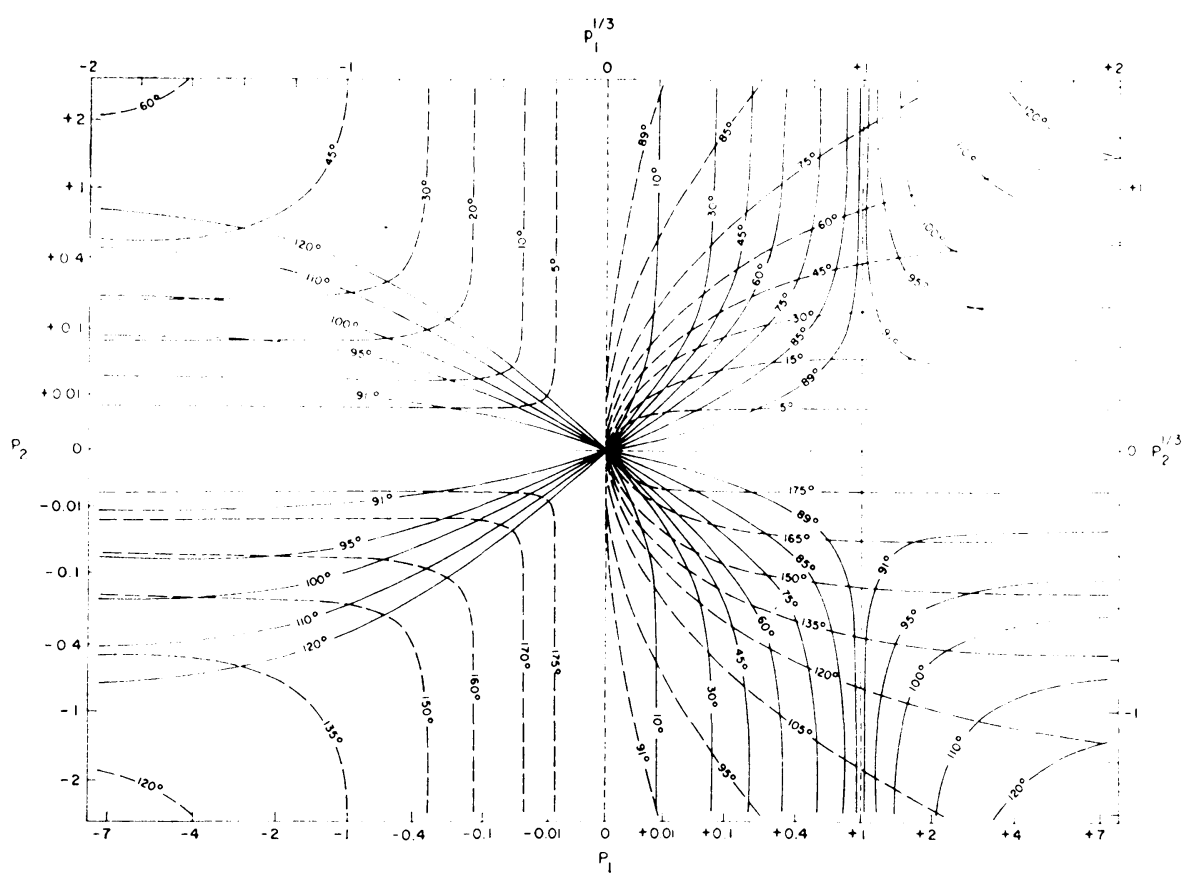

Fig. 1. Relative amplitudes $p_{1}, p_{2}$ of the periodic terms of the radial and transverse components, respectively, of the nongravitational acceleration in terms of the lag angle and obliquity. Solid curves are lines of constant lag angle; broken curves are lines of constant obliquity. Dotted lines run through the loci of singular solutions. The axes of abscissas and ordinates give, on a linear scale, the cube roots of $p_{1}$ and $p_{2}$, respectively. 
transverse-to-radial component ratio $A_{2} / A_{1}$. The conclusions predicted from the analysis can be summarized thus:

(1) If the lag angle $\Lambda$ is small, the radial component of the nongravitational acceleration is positive and well determined, and the $A_{2} / A_{1}$ ratio is small.

(2) If the lag angle is near to or in excess of $90^{\circ}$, the radial component can come out either positive or negative, and it is poorly determined. The transverse component $A_{2}$ may become comparable with $A_{1}$ in magnitude.

(3) The determinacy of $A_{2}$ is almost independent of the lag angle, and very good unless the obliquity $\varepsilon$ approaches $90^{\circ}$.

(4) Positive $A_{2}$ does not necessarily refer to a direct sense of rotation, and vice versa. For obliquities near $90^{\circ}$, the sign of $A_{2}$ can be contrary to the expected one.

(5) The determinacy of $A_{3}$ is poor independently of $\varepsilon$ and $\Lambda$.

We find that the properties of the empirical coefficients $A_{i}$ of the two groups of shortperiod comets outlined in Section 2 are much like the expected properties of $A_{i}$ for small and large lag angles, respectively. We therefore conclude that the two groups of comets differ from each other in the magnitude of the lag angle. Without bringing forward any argument here we point out that over a long time-scale the age of the comet (i.e., basically the number of revolutions about the Sun in an orbit with fairly small perihelion distance) is suspected to be the dominant influence on the lag angle.

\section{Precession of the Cometary Nucleus. Periodic Comet Faye}

A recent study of the motion of P/Faye between 1843 and 1970 (Marsden and Sekanina, 1971) has resulted in the surprising discovery that this comet had a secular acceleration until quite recently, but a secular deceleration since. The transverse-toradial component ratio turned out to be smaller than for any other comet so far investigated and seems to be subject to variations with a period of about $140 \mathrm{yr}$ (see Figure 2).

The variations can be interpreted as an effect of precession of the comet's nucleus. Two versions are suggested:

(1) Regular precession with the axis of precession perpendicular to the orbit plane. Then

$$
\begin{aligned}
& \varphi=\varphi_{0}+\frac{2 \pi t}{P_{p r}}, \\
& \varepsilon=\text { const },
\end{aligned}
$$

where $P_{p r}$ is the period of precession, equal to double the period of the variations in $A_{2} / A_{1}$. This model gives for $\mathrm{P} /$ Faye:

$$
\begin{aligned}
P_{p r} & =37.8 \text { revolution periods } \\
& =280 \mathrm{yr}, \\
\varphi_{0} & \left.=150^{\circ} \text { or } 330^{\circ} \quad \text { (in } 1843\right), \\
\varepsilon & =88.5, \\
\Lambda & =27.3 .
\end{aligned}
$$




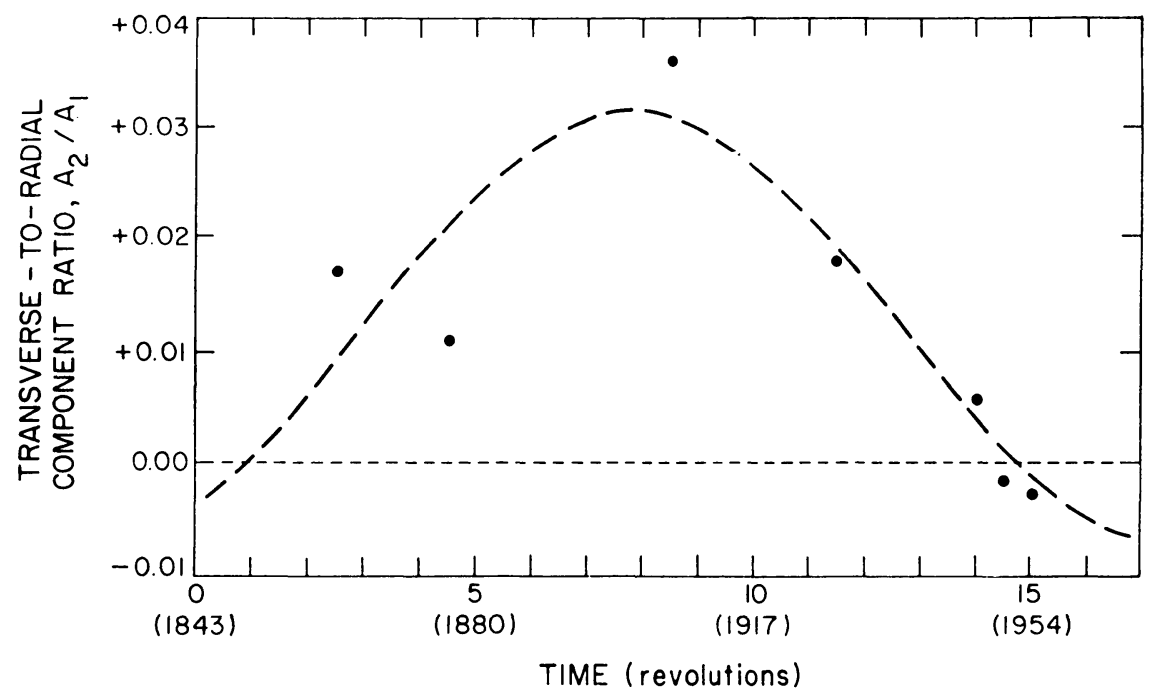

Fig. 2. Secular variations in the transverse-to-radial component ratio for $\mathrm{P} /$ Faye. The points are the empirical data from Marsden and Sekanina (1971). The dashed curve is the best sinusoidal fit; the dotted line is the line of $A_{2}$ sign change. Time is given in both the revolution-period units and years.

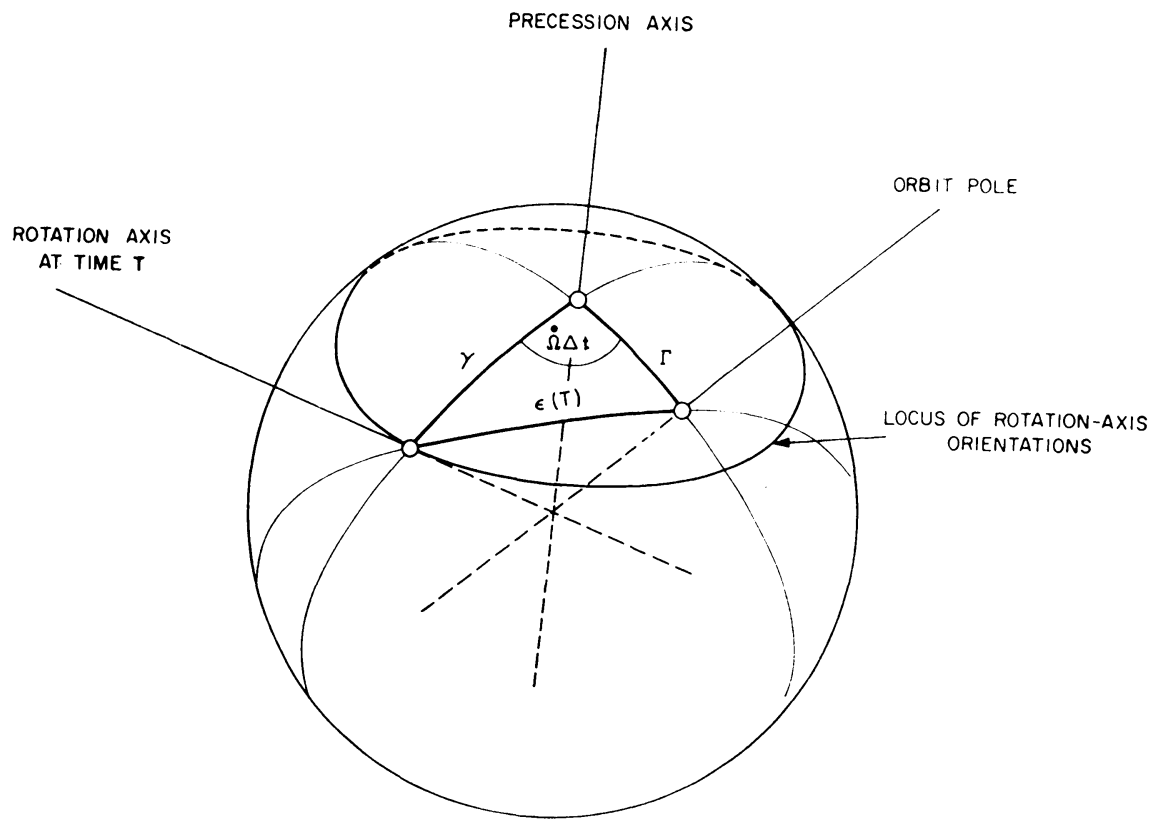

Fig. 3. Instantaneous obliquity $\varepsilon(T)$ in terms of the precession radius $\gamma$, precession axis deviation $I$ and precession angular speed $\dot{\Omega}=2 \pi / P_{p r}$ for the variable-obliquity precession model. We denote $\Delta t=T-t_{0}$, where $t_{0}$ is the time of minimum $\varepsilon$. 
(2) Variable-obliquity precession with the axis of precession tilted to the orbit plane. The obliquity varies with time according to (Figure 3 )

$$
\cos \varepsilon=\cos \gamma \cos \Gamma+\sin \gamma \sin \Gamma \cos \frac{2 \pi\left(t-t_{0}\right)}{P_{p r}}
$$

where $t_{0}$ is the unknown epoch of minimum $\varepsilon ; \varepsilon_{\min }=|\gamma-\Gamma| ; \gamma$ is the precession radius, and $\Gamma$ is the deviation of the precession axis from the orbit pole. The precession period $P_{p r}$ now equals the period of the variations in $A_{2} / A_{1}$. Applied to $\mathrm{P} /$ Faye this model gives:

$$
\begin{aligned}
P_{p r} & =18.9 \text { revolution periods } \\
& =140 \mathrm{yr}, \\
t_{0} & =1902, \\
\tan \gamma \tan \Gamma & =1.52, \\
90^{\circ} & <\gamma+\Gamma \leqq 102^{\circ}, \\
\Lambda & \geqq 1.8 .
\end{aligned}
$$

The angles $\gamma$ and $\Gamma$ cannot be determined unequivocally.

\section{Change in Sign of $A_{2}$ in the Presence of Severe Orbit Transformations: Periodic Comet Pons-Winnecke. A Final Remark}

Marsden (1970) has found that P/Pons-Winnecke was secularly accelerated before 1875 [a result agreeing with that found by Oppolzer (1880)] but slightly decelerated after 1933. A detailed analysis of the motion of this comet suggests that the combined effect of nuclear rotation with a secular increase in the lag angle and of long-term orbit perturbations may be responsible for the change in the sign of $A_{2}$. The result is, however, rather uncertain numerically, because the comet is dynamically troublesome (regular close encounters with Jupiter). Apparently, the comet rotates in a retrograde sense $\left(95^{\circ}<\varepsilon<100^{\circ}\right)$.

The suspicion that $\mathrm{P} / \mathrm{Faye}$ and $\mathrm{P} / \mathrm{Pons}-\mathrm{Winnecke}$ may have rotation axes almost in their orbit planes may be significant; from completely independent evidence we suggested earlier that this could also be the case with $\mathrm{P} / \mathrm{Halley}$ and some other comets (Sekanina, 1967). Recently Gehrels et al. (1970) have concluded that Icarus has an obliquity of almost $90^{\circ}$. Uranus has an obliquity of nearly $90^{\circ}$ too. The fact that there seem to be quite a few bodies of this sort in the solar system suggests that an effort should be made to discover whether there is a mechanism that could force the rotation axis of a body toward its orbit plane.

\section{References}

Gehrels, T., Roemer, E., Taylor, R. C., and Zellner, B. H.: 1970, Astron. J. 75, 186.

Marsden, B. G.: 1969, Astron. J. 74, 720.

Marsden, B. G.: 1970, Astron. J. 75, 75.

Marsden, B. G.: 1972, this Symposium, p. 135. 
Marsden, B. G. and Sekanina, Z.: 1971, Astron. J. 76, 1135.

Oppolzer, T.: 1880, Astron. Nachr. 97, 149.

Sekanina, Z.: 1967, Bull. Astron. Inst. Czech. 18, 286.

Yeomans, D. K.: 1972a, this Symposium, p. 181.

Yeomans, D. K.: 1972b, this Symposium, p. 187. 